\title{
Effect of Basal Media and Sugar Types on in Vitro Regeneration of Grammatophyllum speciosum Blume
}

\author{
Montakarn PIMSEN, Kamnoon KANCHANAPOOM* \\ Prince of Songkla University, Department of Biology, Faculty of Science, Hat-Yai, Songkhla, \\ 90112, Thailand; kamnoon_k@yahoo.co.th (*correspondingauthor)
}

\begin{abstract}
Protocorms of Grammatophyllum speciosum Blume. were initiated from immature seeds on solid MS medium containing 15\% $(\mathrm{v} / \mathrm{v})$ coconut water $(\mathrm{CW})$ and $3 \%(\mathrm{w} / \mathrm{v})$ sucrose. Protocorms, $2-4 \mathrm{~mm}$ in length were used as explants and subcultured to MS and VW (Vacin and Went, 1949) media containing 15\% (v/v) CW and 2 or 3\% (w/v) sucrose. Protocorms gave the highest formation of PLBs (protocorm-like bodies) at 3.1 PLBs/explant on MS solid medium containing 15\% CW and 2\% sucrose. For the test with different carbon sources, protocorms were cultured in liquid MS medium supplemented with 4 kinds of sugar, namely sucrose, glucose, sorbitol and mannitol at 2, 4, 6 or $8 \%(\mathrm{w} / \mathrm{v})$ and cultured for 1, 2, 3 or 4 weeks. They were then transferred to MS solid medium containing $15 \%$ $\mathrm{CW}$ and 2\% sucrose. Results revealed that after 4 weeks in MS liquid medium, sucrose and glucose had an inhibitory effect and $8 \%$ glucose gave a high percentage of protocorm browning. In contrast, sorbitol and mannitol were effective for protocorm regeneration and both sugar alcohols had a positive effect on the formation of PLBs and the development of PLBs into plantlets.
\end{abstract}

Keywords: carbon sources, protocorms, regeneration, tiger orchid

\section{Introduction}

The genus Grammatophyllum contains twelve species. Grammatophyllum speciosum is the only species of the genus Grammatophyllum that is native to Thailand (Sopalun et al., 2010). Its major distribution regions range from South East Asia to New Guinea. This orchid is also known as the tiger orchid or giant orchid. Its flower is yellow with dark red spots and can remain in bloom for 2 months. It is valued for its attractive flowers and used in traditional medicine in Thailand (Sopalun et al., 2010).

Micropropagation of the orchid through formation of PLBs has been reported using various kinds of explants such as shoot tips, axillary buds, flower stalk buds and protocorms (Tokuhara and Mii, 2003). The protocorm is an early stage in the germination of the orchid seed. It is round or oval in shape with a root and shoot meristem that has the capacity to develop into plantlets directly (Chang et al., 2005). Sugar is a very important component in the culture medium as a carbon source because of the low $\mathrm{CO}_{2}$ concentration present in in vitro conditions. An appropriate type and concentration of sugar is needed to promote seed germination and regeneration of plant growth (Faria et al., 2004). Sugars have essential roles acting in a hormone-like manner to affect photosynthesis, respiration and metabolic activity (Rolland et al., 2002). In this paper, we studied the basal media and different sugar types required to induce PLBs from protocorms and the subsequent regeneration of $G$. speciosum.

\section{Material and methods}

\section{Effect of media}

Protocorms about 2-4 $\mathrm{mm}$ in length were cultured on MS and VW supplemented with $15 \%$ (v/v) coconut water $(\mathrm{CW})$ and 2 or $3 \%(\mathrm{w} / \mathrm{v})$ sucrose. The $\mathrm{pH}$ of the medium was adjusted to 5.5 before adding $8 \mathrm{~g} / \mathrm{l}$ agar. The culture vessels were incubated at $25 \pm 1^{\circ} \mathrm{C}$ with a $16 \mathrm{~h}$ photoperiod. The formation of PLBs was determined after 4 weeks of culture. This part resulted in a medium that was suitable for the induction of PLBs.

\section{Effect of types and concentrations of sugar}

Protocorms were cultured on MS (Murashige and Skoog, 1962) liquid media supplemented with $15 \% \mathrm{CW}$ and several sugars namely sucrose, glucose, sorbitol and mannitol at concentrations of $0,2,4,6$ or $8 \%(\mathrm{w} / \mathrm{v})$ in $2 \mathrm{ml}$ eppendorff tubes and incubated at $25 \pm 1^{\circ} \mathrm{C}$ with a $16 \mathrm{~h}$ photoperiod for 1, 2, 3 or 4 weeks. After each period, protocorms were transferred to the induction medium and the data were collected by counting the surviving protocorms and PLBs.

\section{Results and discussion}

\section{Effect of media}

After 4 weeks of culture of G. speciosum protocorms on MS solid medium containing $15 \%(\mathrm{v} / \mathrm{v})$ coconut water and $2 \%(\mathrm{w} / \mathrm{v})$ sucrose most of the protocorms had a 


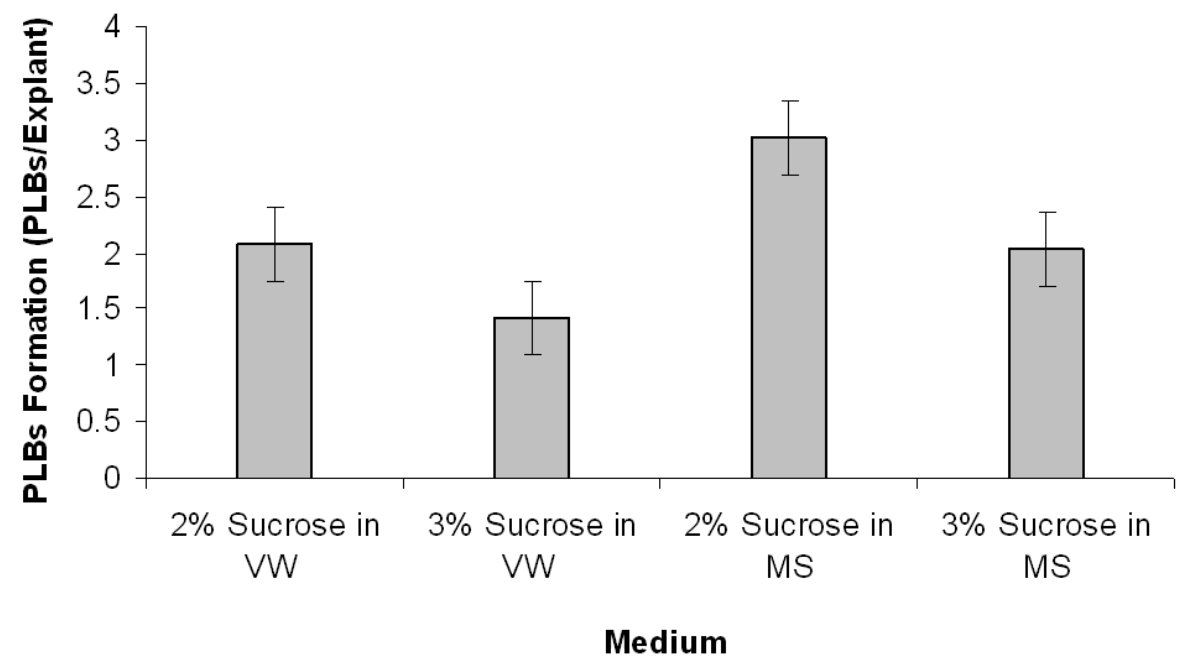

Fig. 1. Formation of PLBs after 4 weeks in media with different basal medium and concentrations of sucrose. All media contained $15 \%(\mathrm{v} / \mathrm{v})$ of coconut water. In each treatment 100 explants were studied. Bar $=$ standard error

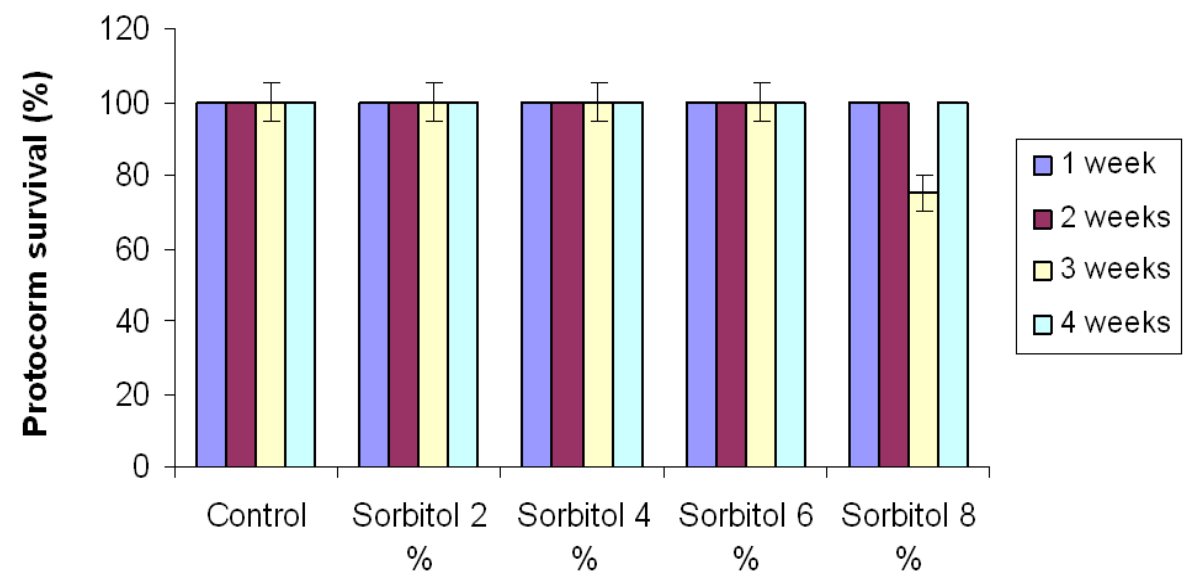

A

Carbon sources

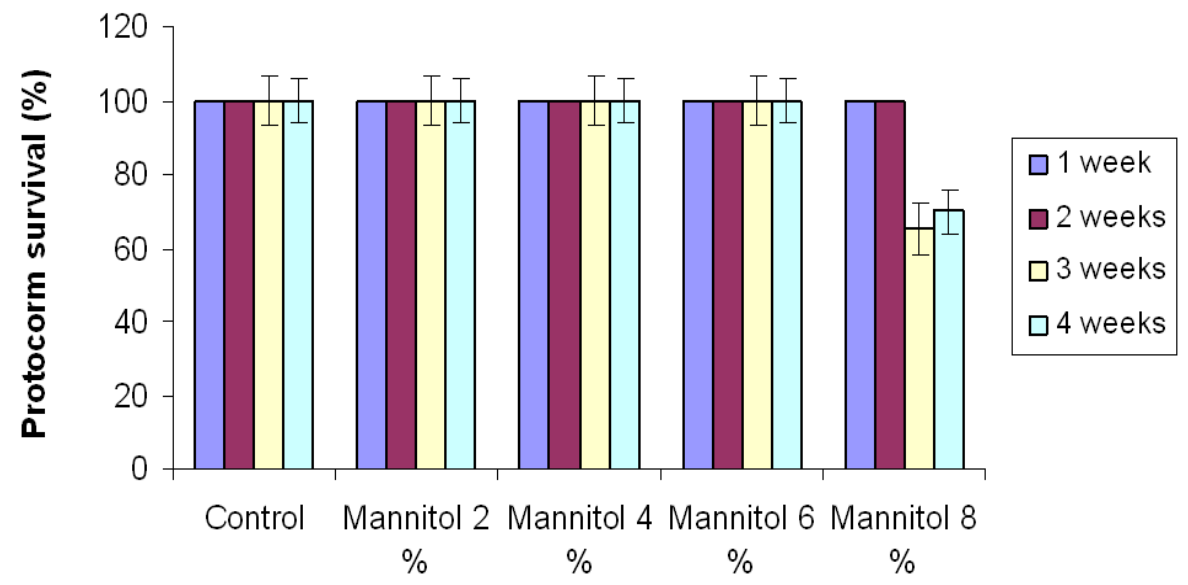

B

Carbon sources

Fig. 2. Regeneration of protocorms after 4 weeks in media with different types and concentrations of sugar. In each treatment 20 explants were studied. Bar = standard error 


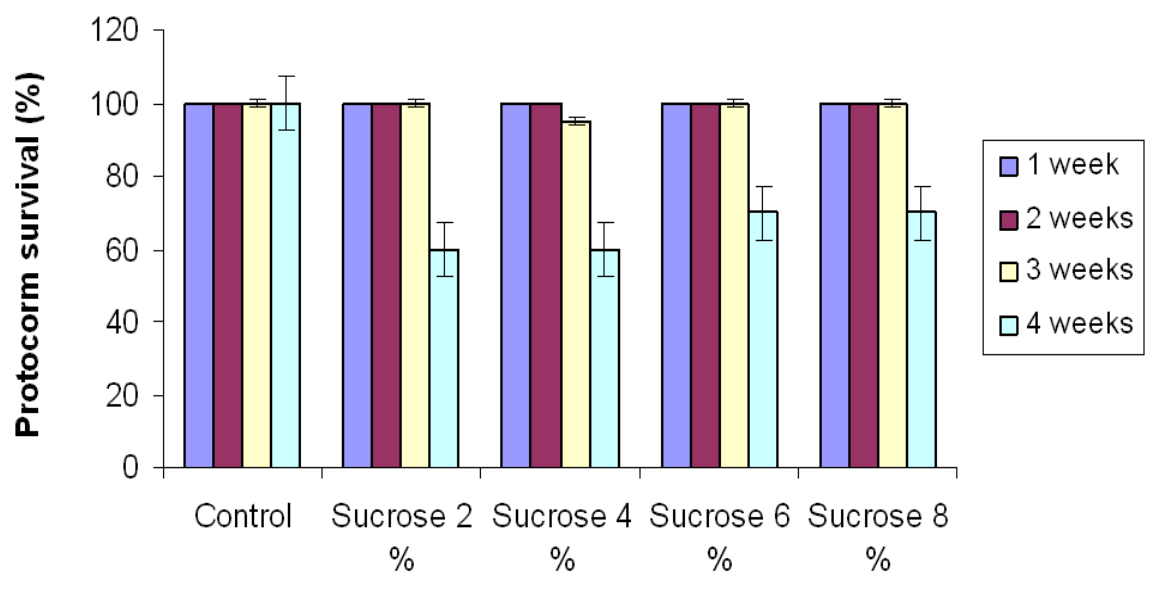

C

\section{Carbon sources}

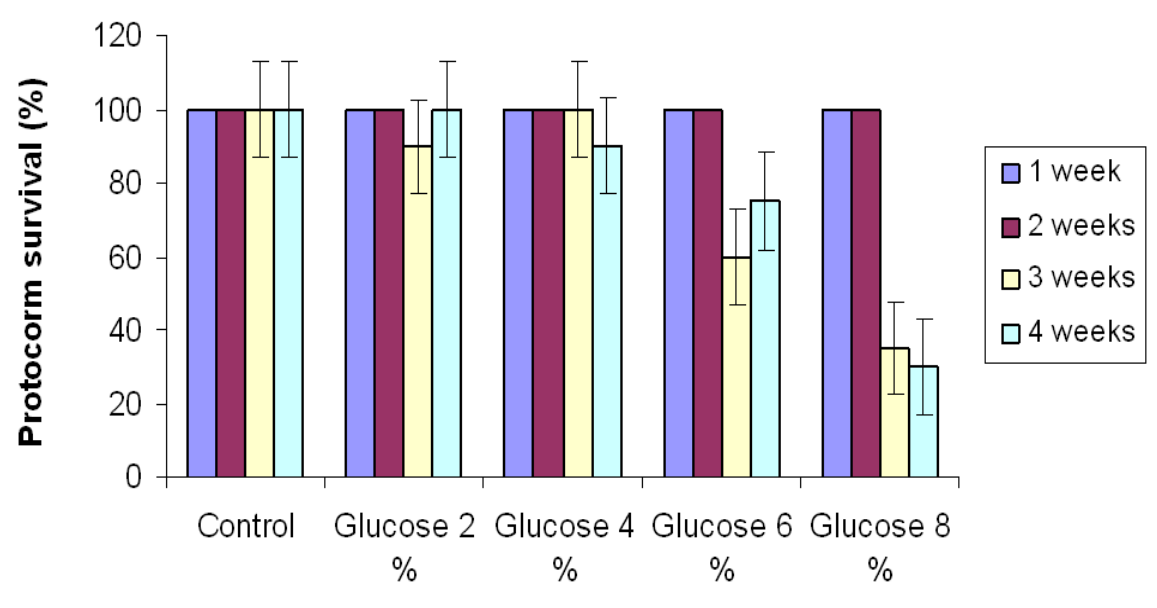

D

\section{Carbon sources}

Fig. 2. Regeneration of protocorms after 4 weeks in media with different types and concentrations of sugar. In each treatment 20 explants were studied. Bar = standard error

high survival rate (84\%) and produced PLBs (3.1 PLBs/ explant). The lowest formation of PLBs (1.46 PLBs/explant) was found when cultured on VW medium containing $15 \% \mathrm{CW}$ and $3 \%$ sucrose (Fig. 1). This result showed that MS medium was suitable for the formation G. speciosum PLBs and was similar to the results reported by Sopalun et al. (2010) that shoot tips of G. speciosum cultured on three different basal media including VW, MS and $1 / 2$ MS produced the highest survival rates of $23 \%$. For Vanda dearie, growth of the protocorms was superior in $1 / 2 \mathrm{MS}$ medium followed by KC and VW media with the highest percentage of protocorms producing leaves $(65 \%)$ and roots (21.67\%) (Jawan et al., 2010). Seeds of Satyrium nepalense were germinated on MS, KC and modified KC media and the highest percentage of seed germination was observed on MS medium (86.7\%) followed by modified $\mathrm{KC}$ and KC (Mahedran and Narmatha, 2009).

\section{Effect of types and concentrations of sugar}

Fig. 2 shows the effect of different types and concentrations of sugar on regeneration of protocorms, MS medium supplemented either with mannitol or sorbitol gave $100 \%$ regeneration of green protocorms and produced PLBs (Fig. 2A, B). Sucrose and glucose had an inhibitory effect on the growth of protocorms and $8 \%$ glucose gave the lowest survival of protocorms (30\%) (Fig. 2D) when most of the protocorms turned brown and did not regenerate to plantlets. The results have shown that different carbon sources and concentrations had a significant effect on the regeneration of plants, which is in agreement with the finding of Hong et al. (2007). They cultured plantlets derived from leaf somatic embryos of Oncidium on hormone-free $1 / 2$ MS medium supplemented with 5 kinds of sugars, and showed that cellobiose had an inhibitory effect but fructose, glucose, sucrose and maltose were all effective for the direct induction of embryos. Tokuhara and Mii 
104

(2003) tested carbohydrate sources and concentrations on the formation of PLBs from cell suspensions of the Phalaenopsis orchid and they reported that glucose was the best carbohydrate source for producing PLBs, sucrose was suitable for callus proliferation and sorbitol for growth of PLBs. Therefore the development of PLBs from cell suspensions of Phalaenopsis could be controlled by changing the types and concentrations of sugars.

In summary, this study has shown that the growth of $G$. speciosum protocorms was enhanced by MS medium containing sorbitol and mannitol. Higher concentrations of glucose and sucrose had an inhibitory effect.

\section{Acknowledgements}

This study was financially supported by Prince of Songkla University Graduate Studies Grant.

\section{References}

Chang C, Chen YC, Yen HF (2005). Protocorm or rhizome? The morphology of seed germination in Cymbidium dayanum Reichb. Bot Bull Acad Sinica 46:71-74.

Faria RT, Rodrigues FN, Oliveira LVR, Muller C (2004). In vitro Dendrobium nobile plant growth and rooting in different sucrose concentrations. Hort Brasileira 22:780-783.
Jawan R, Gansau JA, Abdullah JO (2010). In vitro culture of Borneo's endemic orchid, Vanda dearei. Asia-Pacific J Molec Biol Biotechnol 18(1):203-207.

Hong PI, Chen JT, Chang WC (2007). Promotion of direct somatic embryogenesis of Oncidium by adjusting carbon sources. Biol Plant 52(3):597-600.

Mahedran G, Narmatha BV (2009). Mass propagation of Satyrium nepalense D. Don. A medicinal orchid via seed culture. Sci Hort 119:203-207.

Murashige T, Skoog F (1962). A revised medium for rapid growth and bioassays with tobacco tissue cultures. Physiol Plant 15:473-497.

Rolland F, Moore B, Sheen J (2002). Sugar sensing and signaling in plants. The Plant Cell 14:185-205.

Sopalun S, Thammasiri K, Ishikawa K (2010). Micropropagation of the Thai orchid Grammatophyllum speciosum Blume. Plant Cell Tissue Organ Cult 101:143-150.

Tokuhara K, Mii M (2003). Highly-efficient somatic embryogenesis from cell suspension cultures of Phalaenopsis Orchid by adjusting carbohydrate sources. In Vitro Cell Develop Biol Plant 39:635-639.

Vacin $\mathrm{E}$, Went $\mathrm{F}$ (1949). Some $\mathrm{pH}$ changes in nutrient solution. Bot Gazette 110:605-613. 